\title{
Daytime of sampling, tooth-brushing and ascorbic acid influence salivary thiobarbituric acid reacting substances - A potential clinical marker of gingival status
}

\author{
Július Hodosy ${ }^{\mathrm{a}}$ and Peter Celec ${ }^{\mathrm{b}, *}$ \\ ${ }^{a}$ Institute of Physiology, Faculty of Medicine, and Department of Animal Physiology and Ethology, Faculty of \\ Natural Sciences, Comenius University, Bratislava, Slovak republic \\ ${ }^{\mathrm{b}}$ Institute of Pathophysiology, Faculty of Medicine, and Department of Molecular Biology, Faculty of Natural \\ Sciences, Comenius University, Bratislava, Slovak republic
}

\begin{abstract}
Background. Salivary thiobarbituric acid reacting substances (TBARS) have been previously shown to correlate with the impairment of gingival tissue. Although the details on the origin and the composition of this heterogeneous group of compounds in saliva are unknown, the potential clinical usefulness makes necessary the studies of factors influencing the salivary TBARS levels.

Aim. To observe the effects of daily dynamics, tooth-brushing and ascorbic acid administration on salivary TBARS levels. Subjects and methods. Self-collected samples were obtained from 10 young healthy men collecting samples in the morning, in the afternoon and in the evening during 2 consecutive days. Ascorbic acid $(250 \mathrm{mg})$ was administered orally after the last sampling on day 1 and before every sampling on day 2. Additional sampling was performed before and after tooth-brushing. TBARS levels in saliva specimens were detected spectrofluorometrically. Sialic acid content was measured using a modified method of Warren.

Results. Salivary TBARS levels vary significantly during a day $(p<0.001)$ with highest concentrations in the morning. Both, tooth-brushing $(p<0.05)$ and short-term antioxidative treatment with ascorbic acid $(p<0.005)$ decrease salivary TBARS levels. Sialic acid content of saliva is not influenced significantly by any of the investigated factors.

Conclusion. TBARS levels in saliva are affected by daytime of sampling, tooth-brushing and ascorbic acid pre-treatment. These results must be considered in clinical research using salivary TBARS levels. Sialic acid seems not to be a major component of TBARS in saliva. Further studies should clarify the molecular compounds of salivary TBARS and uncover the role of oral microbial factors.
\end{abstract}

Keywords: Thiobarbituric acid reacting substances, malondialdehyde, sialic acid, ascorbic acid, tooth-brushing

\section{Introduction}

Inflammatory diseases of the gingival tissue have been previously associated with increased oxidative

${ }^{*}$ Corresponding author: Peter Celec, MD, Dipl. Ing., BSc., PhD, Galbavého3, 84101 Bratislava, Slovakia. E-mail: petercelec@gmail. com; URL: www.biomed.szm.com. stress (OS). This assumption was proved using measurement of markers of OS in direct tissue specimens [5], which are quite difficult to obtain and, in addition, it is an invasive procedure for the patient. In a previous study, we have shown that markers of OS can be measured in saliva [3]. The level of thiobarbituric acid reacting substances (TBARS) correlates with the papillary bleeding index (PBI) and could be used clin- 
ically as a simple and non-invasive marker of gingival status [4].

However, there are major problems yet to solve. We still do not know the origin of TBARS in saliva. Although diffusion from plasma seems not to influence the salivary levels of TBARS considerably [6], the site of their production remains unknown. Some indices point towards microbial flora, but also immune system activation and especially cell-mediated immune reaction are known to increase reactive oxygen species (ROS) concentrations [11].

Most clinical biochemical parameters have a number of pitfalls that are, unfortunately, unknown to the users - both, the patients and the doctors. To these pitfalls belong external influences that significantly influence the results and endogenous chronobiological rhythms, which often cause considerable timedependent variations of the parameters. Previous report on a circadian rhythm of antioxidative status [9] forced us to look at the possible daily variations of salivary TBARS. According to our knowledge, neither antioxidative treatment nor tooth-brushing were previously investigated on the effects on salivary TBARS. Thus, the aim of this study was to look for daily variations and the short-term effects of ascorbic acid or tooth-brushing on salivary TBARS levels.

\section{Subjects and methods}

\subsection{Design}

10 young healthy male volunteers (aged $21.8 \pm 1.8$ years) were asked to collect salivary samples during two consecutive days. All probands participated already on our previous studies and thus understood the details of the procedure. Moreover, the results of biochemical analyses were similar to probands within the lowest PBI quartile in our previous clinical study. Sampling was performed at 08:00, 14:00 and 20:00 on both days without stimulation. An additional morning sampling was carried out 10 minutes after tooth-brushing. The volunteers were instructed not to eat or drink $30 \mathrm{~min}$ utes before any sampling and were allowed to brush their teeth twice daily (between the two morning sampling times and after the evening sampling). Ascorbic acid was administered orally using effervescent tablets at a dose of $250 \mathrm{mg}$ in the evening (day 1) after the last sampling and before every sampling (at least 30 minutes) on day 2. The volunteers were asked to flush their mouths with the sparkling fluid for one minute and then swallow it.

\subsection{Biochemical analysis}

Collected saliva samples were frozen $\left(-20^{\circ} \mathrm{C}\right)$ until measurement. Salivary TBARS were determined by the spectrofluorometric method $\left(\lambda_{\text {ex. }}=535 \mathrm{~nm}\right.$, $\left.\lambda_{\text {em. }}=553 \mathrm{~nm}\right)$ after derivatization with $0,6 \%$ thiobarbituric acid in acidic medium of acetic acid $\left(100^{\circ} \mathrm{C}\right.$, 45 min.). After derivatization, the coloured product was extracted to n-butanol, centrifuged $(3000 \mathrm{rpm}$, 10 min.) and measured $[13,18]$. The calibration curve was calculated using 1,1,3,3-tetrametoxypropan as a malondialdehyde standard. TBARS concentrations were expressed in $\mu \mathrm{mol} / 1$. Sialic acid was measured using a modified method by Warren [2,7,19]. Due to missing sialic acid standard, determined concentrations are expressed in arbitrary units (AU).

\subsection{Statistical analysis}

Standard nonparametric Wilcoxon test for two related samples and Friedman test for more related samples were used (both tests at the significance level $\alpha=0.05$ ). Analysis of the results was performed with Microsoft ${ }^{\mathrm{TM}}$ Excel $2000{ }^{\circledR}$ software, XLStatistics 5.51, Micrococal $^{\mathrm{TM}}$ Origin ${ }^{\circledR} 6.0$ and SPSS for Windows 11.0 .

\section{Results}

Although the gingival status of our probands was not assessed in this study, the overall relatively low levels of salivary TBARS indicates a low PBI, if compared to our previous clinical study [4]. The daily variations of salivary TBARS levels, the effect of tooth-brushing and ascorbic acid administration, as well as the dynamics of sialic acid levels are shown on Figs 1-4.

\section{Discussion}

Salivary TBARS are a useful marker of gingival status. However, before the introduction of this parameter into the clinical stomatological use, important issues must be solved. Especially, factors influencing the levels of salivary TBARS should be examined. We have decided to prove some of these possible factors. 


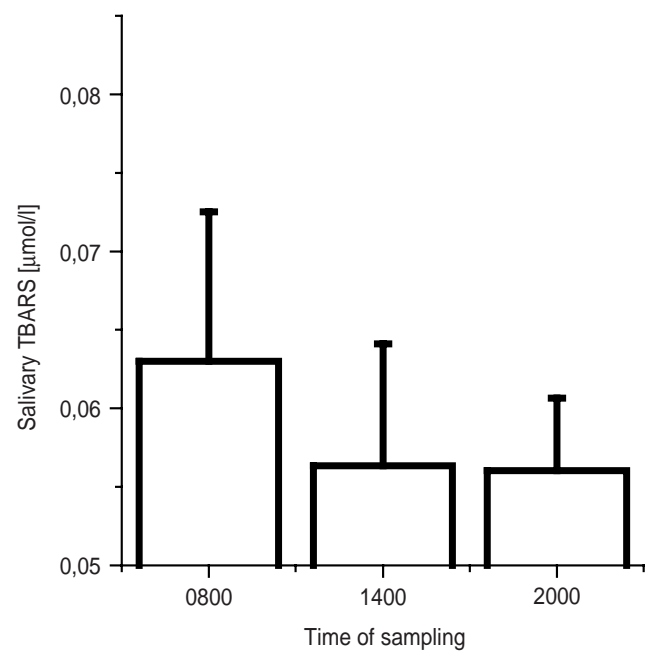

Fig. 1. Daily variations of salivary TBARS. Note the considerable decrease between the morning and afternoon samplings $\left(\chi^{2}=13.56 ; p<0.001\right)$. The results are presented as mean + standard deviation.

\subsection{Daily variation}

As seen on Fig. 1 salivary TBARS exhibit a significant daily variation. Of the three sampling times morning levels are clearly the highest. Detailed analysis of the possible circadian rhythm in the future is needed. Of course, night samples will be difficult to obtain. The nocturnal dynamics is more or less of theoretical value, as most clinical sampling will be done during the day. The daily variations on the other hand are very important for the understanding and interpretation of TBARS levels in the clinical use. Further research should concentrate on a question how TBARS dynamics affects the relationship of salivary TBARS and PBI.

\subsection{Tooth-brushing}

Cleaning the teeth decreases salivary TBARS levels (Fig. 2). This result is not surprising. As TBARS are of local oral origin, tooth-brushing cleaves the oral cavity and TBARS containing saliva is replaced by fresh blank saliva virtually without TBARS [17]. Most patients coming to a stomatologist clean their teeth straight before the visit. Thus, the previously found correlation expects saliva samples after tooth-brushing. This further stimulates our effort to uncover the real origin of salivary TBARS.

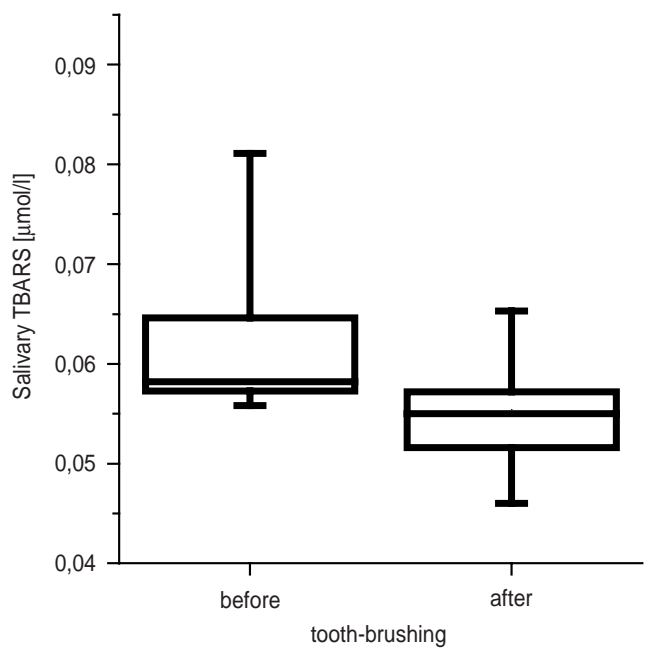

Fig. 2. Effect of tooth-brushing on salivary TBARS levels. Note the significant decrease of salivary TBARS after tooth-brushing $(Z=-1.96, p<0.05)$. The results are presented as 5., 25., 50., 75. and 95. percentile.

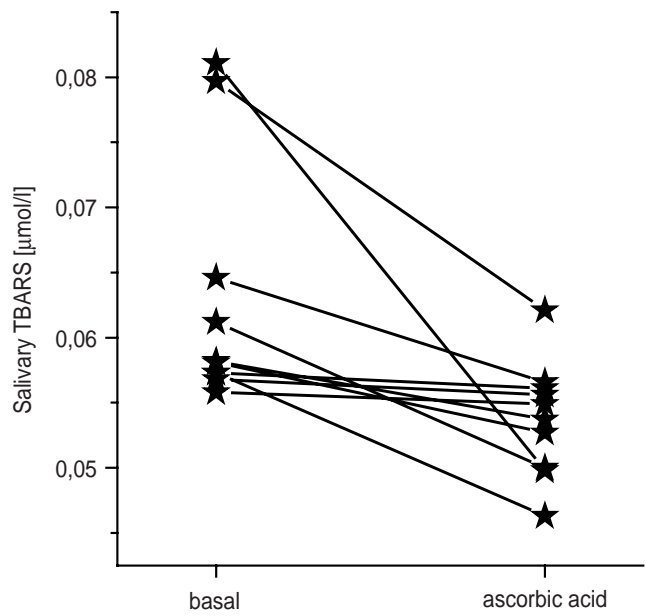

Fig. 3. Effect of ascorbic acid administration on salivary TBARS levels. Ascorbic acid induced a significant decrease of TBARS $(Z=-2.81 ; p<0.005)$. Note that especially volunteers with higher TBARS levels responded to the ascorbic acid antioxidative treatment.

\subsection{Ascorbic acid}

As TBARS are accepted and widely used markers of lipoperoxidation [12], external antioxidative treatment might influence the salivary TBARS levels [10]. The effect of ascorbic acid (Fig. 3) proved that salivary TBARS are a marker of local oxidative stress similar to other biological specimens and tissues. On Fig. 3 the comparison between the morning levels on day 1 (basal values) and the morning levels on day 2 (after 


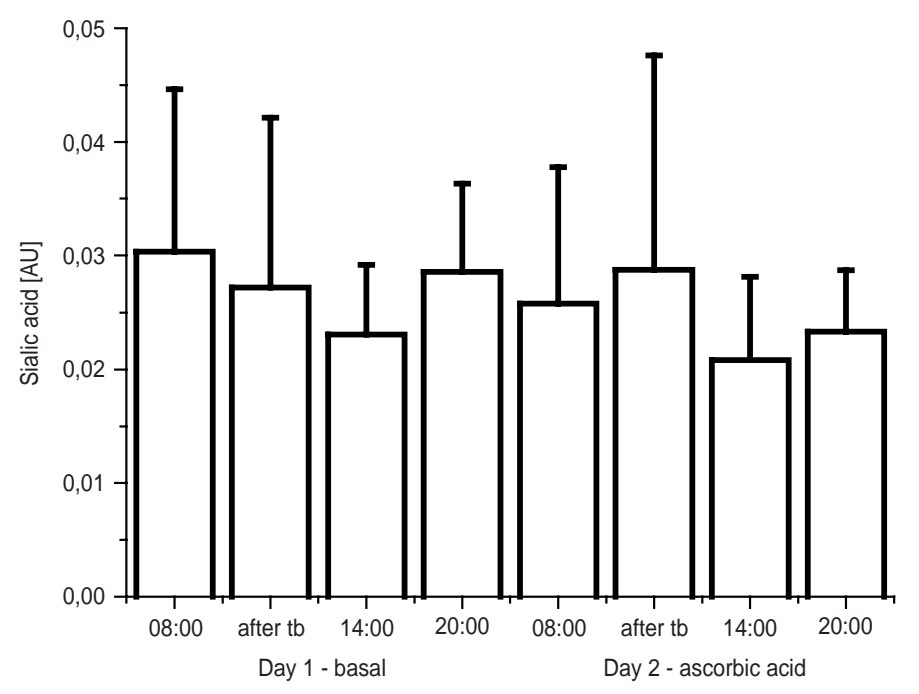

Fig. 4. The dynamics of salivary sialic acid levels. Although there seems to be a trend to reduction of sialic acid concentrations due to ascorbic acid treatment, no significant changes have been found $\left(\chi^{2}=1.75 ; p<0.42\right)$. The results are presented as mean + standard deviation.

ascorbic acid administration) can be seen. Induced decrease at 14:00 and 20:00 was slight and did not reach the level of significance. The main arising question is what could be the effects of long-term antioxidative treatment. There are numerous studies showing a clear benefit of antioxidants on gingival diseases [1,10,1416]. Whether this therapy also durably influences the salivary TBARS levels is unknown.

\subsection{Sialic acid}

No changes have been observed in salivary sialic acid levels during the experiment. It could be expected that at least tooth-brushing might affect the levels, as sialic acid is a sugar on the surface of cells and cleaning the teeth might influence the concentration of salivary epithelial cells torn out from the mucous. But this was not the case. Sialic acid is thus a more stable parameter than TBARS, however, the salivary concentrations of sialic acid have not been previously proved for correlations with gingival status.

TBARS are a group of heterogeneous substances [8]. Despite the fact that TBARS are widely used for measurement of malondialdehyde (MDA), our previous results showed that only about $50 \%$ of salivary TBARS could be accounted to MDA [4]. Thus, in saliva TBARS seem to be composed to a considerable part of other substances. Previous reports about chemical analysis of TBARS suggest other aldehydes to react with the thiobarbituric acid [7]. Especially 9-hydroxy-nonenal as a further product of lipoperoxidation seems to be a major component. On the other hand, some studies point towards sialic acid as the source of interference in MDA measurement using the thiobarbituric acid [12]. Sialic acid is a chemically completely different compound that could make the interpretation and maintenance of TBARS as a marker of OS very difficult. As both factors improving the gingival status, tooth-brushing and ascorbic acid decrease TBARS but do not affect the sialic acid levels, it can be concluded that sialic acid does not make up a substantial part of salivary TBARS, at least not the part that is related to the gingival status. Similar finding eliminated MDA from the list of candidates for salivary TBARS compounds [4]. That is why other molecules should be considered. The decrease, which followed tooth-brushing, supports our hypothesis of local origin. The effect of ascorbic acid reveals the relation of the unknown compound to oxidative stress. What is the mysterious element? A product of oxidative stress induced tissue impairment other than MDA seems thus to be the answer. Hydroxynonenal and prostaglandin endoperoxide could be the plausible candidates. However, other molecules, previously not considered, should be proved in further research. Moreover, the role of local oral microbial diversity as a potential producer of salivary TBARS (or inducer of their production) must be evaluated.

In conclusion, our study shows that salivary TBARS undergo a significant daily variation with highest levels in the morning. Short-term ascorbic acid treatment and tooth-brushing decrease the salivary TBARS levels, while sialic acid levels in saliva are not affected. 
Further studies are needed to determine the major compound of salivary TBARS and to uncover the role of oral microbial flora in salivary TBARS production.

\section{Acknowledgment}

The authors are grateful to the participants, to Assoc. Prof. Lukáč Halčák, to BiomeD research and publishing group for help with biochemical analysis and to Children's Hope Club for continuous moral support. The authors are supported by grant from the Grant Agency for Science and Technology APVT-20003-104.

\section{References}

[1] M. Battino, M.S. Ferreiro, I. Gallardo, H.N. Newman and P. Bullon, The antioxidant capacity of saliva, J Clin Periodontol 29 (2002), 189-194.

[2] V.P. Bhavanandan, N.J. Ringler and D.C. Gowda, Identification of the glycosidically bound sialic acid in mucin glycoproteins that reacts as "free sialic acid" in the Warren assay, Glycobiology 8 (1998), 1077-1086.

[3] P. Celec, T. Červenka, J. Hodosy, P. Boor, S. Veselá, L. Halčák, D. Ostaníková, A. Tomandlová, V. Rendeková and J. Podhradsky, Thiobarbituric acid reacting substances and their relation to the gingival inflammation, Timisoara Med J 54 (2004), 81-85.

[4] P. Celec, J. Hodosy, V. Celecová, J. Vodrážka, L. Halčák, P. Božek, M. Kopáni and M. Kúdela, Salivary thiobarbituric acid reacting substances and malondialdehyde - their relationship to parodontal status, Disease Markers 21 (2005), 133-137.

[5] I.L. Chapple, Reactive oxygen species and antioxidants in inflammatory diseases, J Clin Periodontol 24 (1997), 287296.

[6] J. Hodosy, P. Celec, T. Červenka, J. Vodrážka, S. Veselá, A. Tomandlová, L. Halčák, D. Ostatníková, A. Izakovičová, Z. Putz and P. Božek, Produkty reakcie sliny s kyselinou tiobarbiturovou pri parodontopatiách a ich pôvod, Česká Stomatol 104 (2004), 57-70.
[7] M.E. Gotz, A. Dirr, A. Freyberger, R. Burger and P. Riederer, The thiobarbituric acid assay reflects susceptibility to oxygen induced lipid peroxidation in vitro rather than levels of lipid hydroperoxides in vivo: a methodological approach, Neurochem Int 22 (1993), 255-262.

[8] V.K. Hangloo, I. Kaul and H.U. Zargar, Serum sialic acid levels in healthy individuals, J Postgrad Med 36 (1990), 140-142.

[9] R. Hardeland, A. Coto-Montes and B. Poeggeler, Circadian rhythms, oxidative stress, and antioxidative defense mechanisms, Chronobiol Int 20 (2003), 921-962.

[10] I.G. Holmes, Effects of smoking and/or vitamine C cervical fluid flow in clinically healthy gingiva, Quintessence Int $\mathbf{2 1}$ (1990), 191-195.

[11] H. Katsuragi, M. Ohtake, I. Kurasawa and K. Saito, Intracellular production and extracellular release of oxygen radicals by PMNs and oxidative stress on PMNs during phagocytosis of periodontopathic bacteria, Odontology 91 (2003), 13-18.

[12] D. Lapenna, G. Ciofani, S.D. Pierdomenico, M.A. Giamberardino and F. Cuccurullo, Reaction conditions affecting the relationship between thiobarbituric acid reactivity and lipid peroxides in human plasma, Free Radic Biol Med 31 (2001), 331-335.

[13] G. Lefevre, M. Beljean-Leymarie, F. Beyerle et al., Evaluation of lipid peroxidation by measuring thiobarbituric acid reactive substances, Ann Biol Clin (Paris) 56 (1998), 305-319.

[14] R.M. Nagler, I. Klein, N. Zarzhevsky, N. Drigues and A.Z. Reznick, Characterization of the differentiated antioxidant profile of human saliva, Free Radic Biol Med 32 (2002), 268277.

[15] M. Nishida, S.G. Grossi, R.G. Dunford, A.W. Ho, M. Trevisan and R.J. Genco, Dietary vitamin C and the risk for periodontal disease, J Periodontol 71 (2000), 1215-1223.

[16] D.V. Sculley and S.C. Langley-Evans, Periodontal disease is associated with lower antioxidant capacity in whole saliva and evidence of increased protein oxidation, Clin Sci (Lond) $\mathbf{1 0 5}$ (2003), 167-172.

[17] J.A. Tromp, J. Jansen and T. Pilot, Gingival health and frequency of tooth brushing in the beagle dog model. Clinical findings, J Clin Periodontol 13 (1986), 164-168.

[18] T. Vasankari, U. Kujala, O. Heinonen, J. Kapanen and M. Ahotupa, Measurement of serum lipid peroxidation during exercise using three different methods: diene conjugation, thiobarbituric acid reactive material and fluorescent chromolipids, Clin Chim Acta 234 (1995), 63-69.

[19] L. Warren, Sialic acid in human semen and in the male genital tract, J Clin Invest 38 (1959), 755-761. 


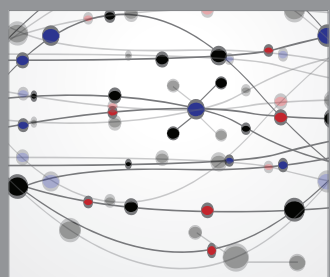

The Scientific World Journal
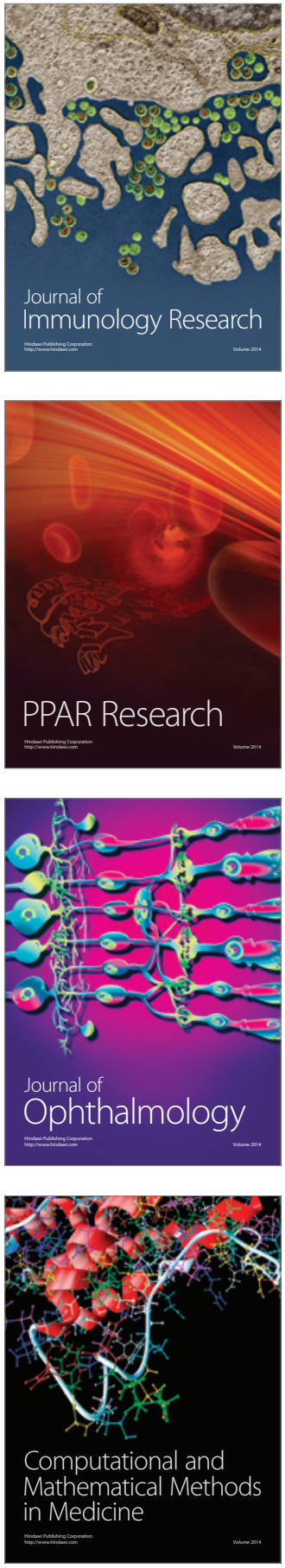

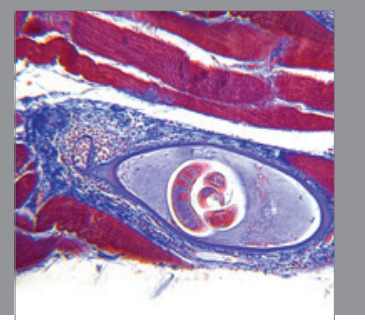

Gastroenterology

Research and Practice
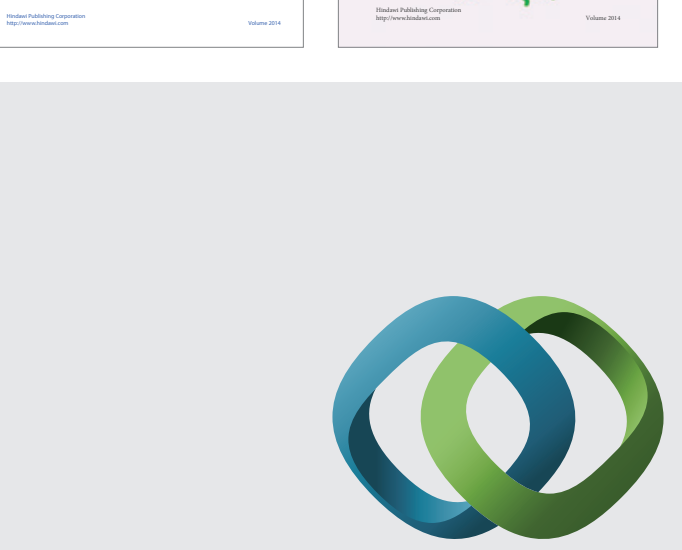

\section{Hindawi}

Submit your manuscripts at

http://www.hindawi.com
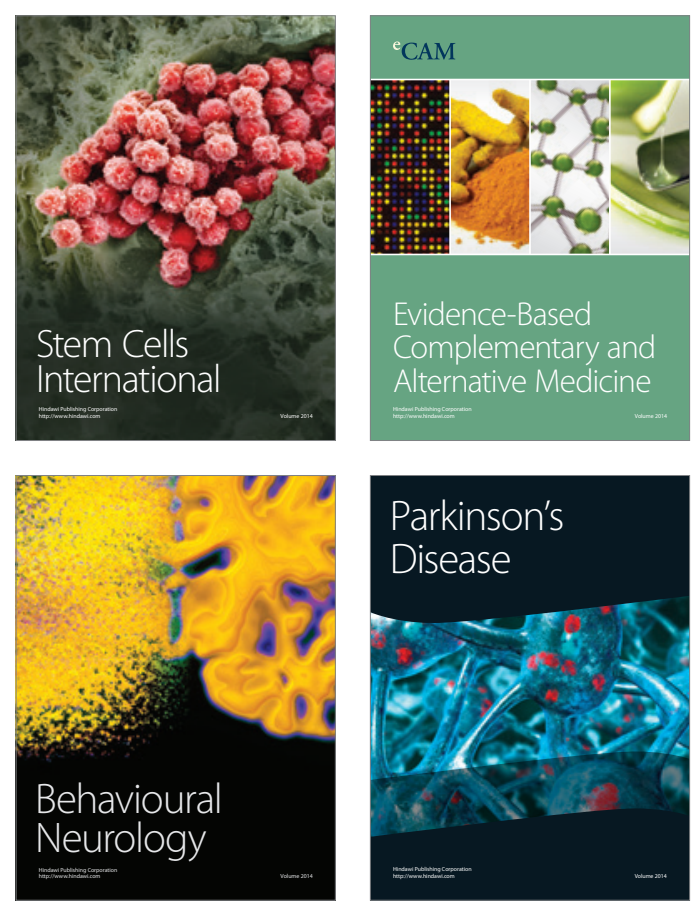

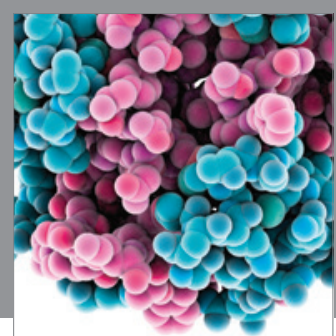

Journal of
Diabetes Research

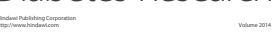

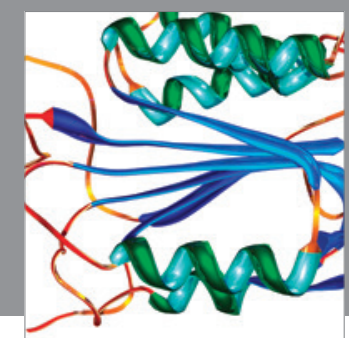

Disease Markers
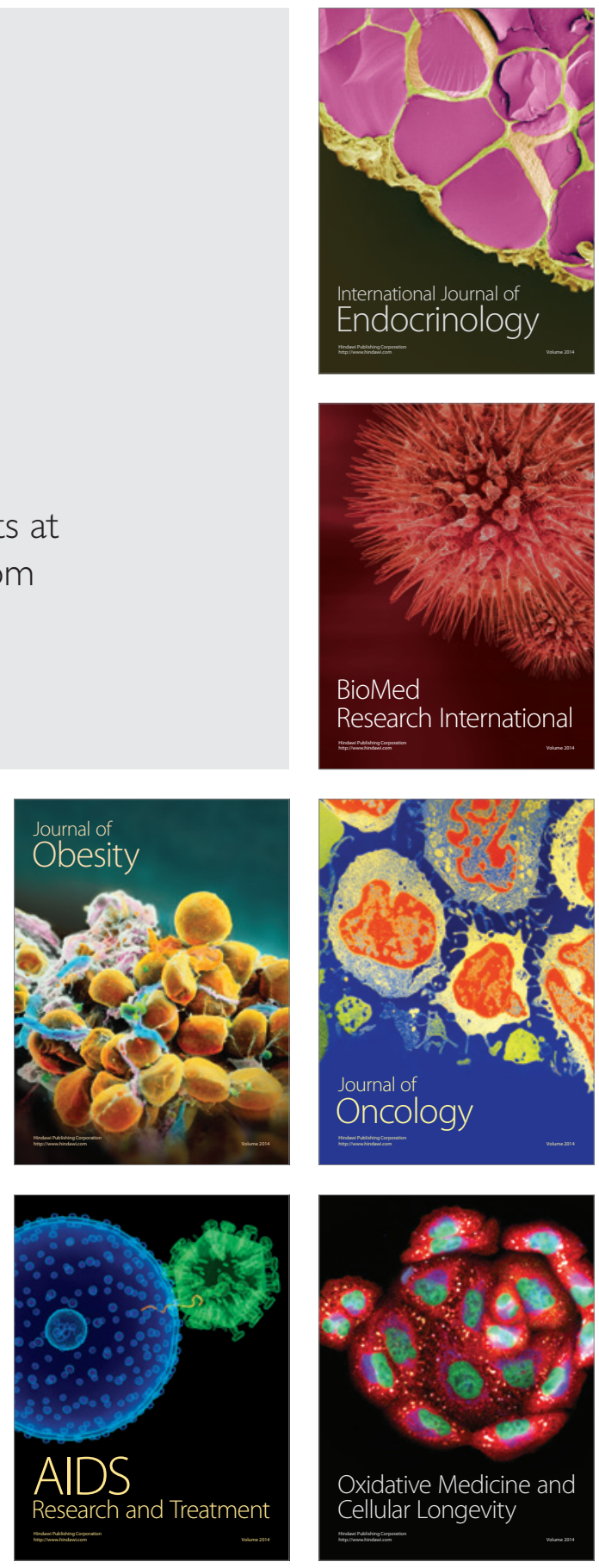